\title{
Studies on $\operatorname{In}_{\mathrm{x}}\left(\mathrm{As}_{2} \mathrm{Se}_{3}\right)_{1-\mathrm{x}}$ thin films using variable angle spectroscopic ellipsometry (VASE)
}

\author{
G.A.M. AMIN* \\ NCRRT, P.O. Box 8029-Nasr City 11371, Cairo, Egypt
}

\begin{abstract}
The results of multi-angle ellipsometrical measurements of thermally evaporated $\operatorname{In}_{x}\left(\mathrm{As}_{2} \mathrm{Se}_{3}\right)_{1-\mathrm{x}}(\mathrm{x}=0,0.01,0.05)$ films are presented. Optical parameters $n$ and $E_{g}$ of thin $\operatorname{In}_{x}\left(\mathrm{As}_{2} \mathrm{Se}_{3}\right)_{1-\mathrm{x}}$ films show that indium atoms were incorporated into the host matrix of $\mathrm{As}_{2} \mathrm{Se}_{3}$ forming distinct features depending on the indium concentration. Refractive index, n, was found to decrease with the addition of In to the binary $\mathrm{As}_{2} \mathrm{Se}_{3}$. The real and imaginary parts of the dielectric function, $\varepsilon^{\prime}$ and $\varepsilon^{\prime \prime}$ were also calculated from the obtained data and correlated with In concentration. It was found that $\varepsilon^{\prime}$ decreases with the increase of In content while $\varepsilon^{\prime \prime}$ increases with the increase of In content. Absorption edge is shifted towards lower photon energy with the increase of In content. As a result, the optical energy gap decreases with increasing In content. This has been correlated with the chemical character of the additive as well as with the structural and bonding aspects of the amorphous composition. Nonlinear optical constants $\left(\chi^{(3)}\right.$ and $\left.\mathrm{n}_{2}\right)$ were determined from linear optical parameters using semi-empirical relations in the long wavelength limit.
\end{abstract}

Keywords: chalcogenides; ellipsometry; optical absorption; energy gap; nonlinear optics

(C) Wroclaw University of Technology.

\section{Introduction}

Chalcogenide glassy semiconductors possess disordered structures with flexible arrangements of the glassy network and the ability to change their optical, physicochemical, and photoelectric properties under external influences and compositional variations [1-4]. One of the most often investigated chalcogenide glasses is the stoichiometric compound $\mathrm{As}_{2} \mathrm{Se}_{3}$, while the non-stoichiometric glasses have received less attention. Due to their potential applications in infrared optics, optoelectronics, optical memories, waveguides, holography, bio- and chemical-sensors, $\mathrm{As}_{\mathrm{x}} \mathrm{Se}_{1-\mathrm{x}}$ glass structures have been intensively studied during recent decades [5]. Chalcogenide glasses exhibit transmittance in a wide spectral range ( 1 to $20 \mu \mathrm{m}$ ), low phonon energy and bandgap, high linear and nonlinear refractive index, and high photo-sensitivity when illuminated by near band gap light [6-8]. Arsenic selenide glasses are heavily studied materials, mainly due to their unique light induced

*E-mail: gamalamin@yahoo.com effects $[9,10]$. Chalcogenide glasses can be deposited as thin film by different techniques as thermal and flash evaporation, spin-coating and pulse laser deposition. As a result, chalcogenide glasses have found practical applications in manufacturing of various elements for optics in visible and infrared spectral region [11], photonics and all optical processing such as waveguides, optics amplifiers, diffraction gratings, microlenses and phase change recording media. To realize all these devices and applications we must know and be able to precisely control optical parameters of these materials. Ellipsometric measurements have been applied for investigation of optical properties of the chalcogenide films and different effects resulting from compositional changes, photo-irradiation and other external factors.

\section{Experimental}

Amorphous $\mathrm{As}_{2} \mathrm{Se}_{3}$ was prepared in bulk form using melt quenching technique. Proper amounts of high purity, 99.999, As and Se elements were placed in a dry clean silica ampoule and then 
evacuated to $1.33 \times 10^{-3} \mathrm{~Pa}$ and sealed under vacuum. The ampoule was placed in a high temperature oven and heated in steps up to $850{ }^{\circ} \mathrm{C}$ and kept at this temperature for 8 hours. Afterwards it was quenched in ice-water mixture to prevent any possible crystallization upon solidification of the liquid. Pure In, $5 \mathrm{~N}$, was added to $\mathrm{As}_{2} \mathrm{Se}_{3}$, in the proper ratios, using the same technique. Prepared bulk material was confirmed to have the desired composition by using an energy dispersive (X-ray) spectroscopy (EDS) unit attached to a Joel scanning electron microscope. Thin films of the prepared bulk material were deposited on glass substrates kept at room temperature under a vacuum of $1.33 \times 10^{-3} \mathrm{~Pa}$ using an Edwards E306 thermal evaporator. Film thickness, which was about $1000 \mathrm{~nm}$, was monitored during evaporation using a crystal oscillator thickness monitor. Composition of the films was confirmed to be the same as that of the bulk material within $\pm 1 \%$. An X-ray diffractometer of Shimadzu XD-DI type was used to confirm the amorphous nature of the grown films. It is clear from Fig. 1 that no sharp peaks, characteristic of the crystalline phase, were observed, confirming the amorphous nature of the films. Ellipsometric measurements were carried out at three angles of light incidence from 45 to $55^{\circ}$ in the photon energy range of $1.9 \mathrm{eV}$ to $4.5 \mathrm{eV}$ with intervals of $0.02 \mathrm{eV}$, using a computer-controlled variable angle spectroscopic ellipsometer, VASE, of the rotating analyzer type from J.A. Woollam Company. The accuracy of the angle of incidence was $\pm 0.005^{\circ}$. Ellipsometry measurements deal with relative changes in amplitude and phase of linearly polarized monochromatic light incident and reflected from a sample surface. Experimentally measured ellipsometry parameters $(\tan \psi$ and $\cos \Delta)$, which are related to the optical and structural properties of the sample, are defined by:

$$
\rho=\frac{R_{p}}{R_{s}}=\tan (\psi) e^{i \Delta}
$$

where, $\rho$ is the complex reflectance ratio, $R_{p}\left(R_{s}\right)$ is the complex reflection coefficient of the light polarized parallel (perpendicular) to the plane of incidence. The two parameters obtained in an ellipsometric measurement are $\psi$ and $\Delta$. From equation 1 , $\psi$ and $\Delta$ can be defined, respectively, by the ratio of the amplitudes of the reflection coefficients for the $\mathrm{p}$ and $\mathrm{s}$ polarized radiation and by the difference in phase between the $\mathrm{p}$ and $\mathrm{s}$ polarized components of the radiation:

$$
\tan (\psi)=\frac{R_{p}}{R_{s}}, \Delta=\delta_{p}-\delta_{s}
$$

In ellipsometry, in general, it is not possible to invert the Fresnel equations to obtain the complex refractive index parameters. Instead, an optical model must be developed to extract the optical parameters. The solution of the reverse problem is to find such values of optical constants and layer thicknesses that provide the best match between the calculated dependencies of $\Psi_{\text {model }}\left(\phi_{\mathrm{i}}, \mathrm{B}\right)$ and $\Delta_{\text {model }}\left(\phi_{i}\right.$, B $)$ using a certain model, and the experimental dependencies of $\Psi \exp \left(\phi_{\mathrm{i}}\right)$ and $\Delta \exp \left(\phi_{\mathrm{i}}\right)$. Here, $B=\left(b_{1}, b_{2}, \ldots b_{m}\right)$ is a parametric vector which characterizes the sample under investigation. The values of parameters for the model used by us were obtained by minimizing the object function:

$$
\begin{aligned}
G(B)= & \sum_{i=1}^{N}\left\{\left(\Psi_{\text {exp }}\left(\phi_{i}\right)-\Psi_{\text {model }}\left(\phi_{i}, B\right)\right)^{2}+\left(\Delta_{\text {exp }}\left(\phi_{i}\right)\right.\right. \\
& \left.\left.-\Delta_{\text {model }}\left(\phi_{i}, B\right)\right)^{2}\right\}
\end{aligned}
$$

Here, $\mathrm{N}$ is the number of angles of incidence. The search of its minimum $\left(\mathrm{G}_{\min }\right)$ was carried out using the modified Nelder-Mead method [12]. To obtain the unique model parameter values, it is necessary to satisfy the $M \gg L$ condition, where $M$ is the number of the experimentally measured ellipsometric parameters, and $\mathrm{L}$ is the quantity of the obtained parameters of the model $[13,14]$. The further complication is that due to the difficulty of the evaluation of the error function, global minimum increases with an increase in the number of the searched parameters. The reasonable course of action in our case is to determine the character of the processes which take place in the films by using the simplest models with the minimal number of parameters. Besides $\mathrm{G}_{\mathrm{min}}$, the important model adequacy evaluation criterion is the physical basis of the obtained solution, and also the match among the properties of thermally evaporated films obtained 
by other independent methods, including those obtained by other authors. Such an approach was realized in the present work. The accuracy of the film thickness assessment was $\pm 0.2 \mathrm{~nm}$, while that of the $\mathrm{N}$ and $\mathrm{k}$ values was \pm 0.005 .

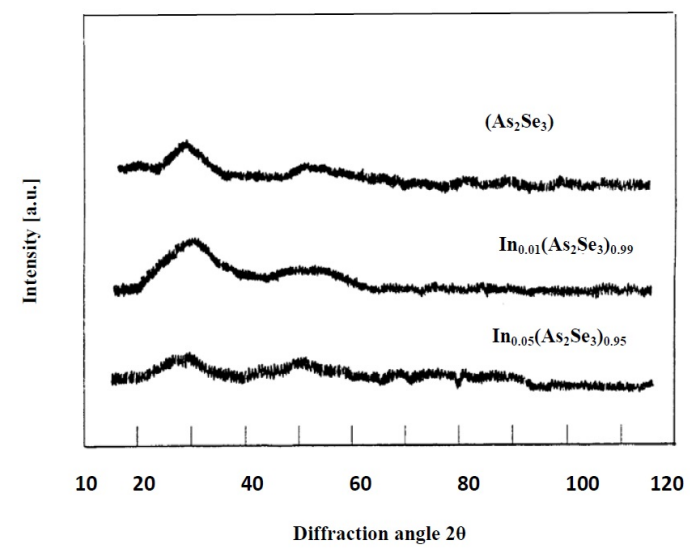

Fig. 1. XRD patterns for the studied amorphous compositions.

\section{Results}

Optical parameters of $\operatorname{In}_{\mathrm{x}}\left(\mathrm{As}_{2} \mathrm{Se}_{3}\right)_{1-\mathrm{x}}$ thin films were determined using a single homogenous absorbing layer model and applying multi-angle measurements $\left(45,50\right.$ and $\left.55^{\circ}\right)$. In this case, the refractive index, $\mathrm{n}$, extinction coefficient, $\mathrm{k}$ and thickness, $d$ were simultaneously calculated (threeparameters of calculation). Fig. 2 shows the spectral dependence of the refractive index for all compositions under investigation. It can be seen that for all films, the refractive index decreases with increasing incident photon energy. Adding In to $\mathrm{As}_{2} \mathrm{Se}_{3}$ leads to decreasing the refractive index in the studied spectral range. On the other hand, the extinction coefficient was found to increase with increasing photon energy for all films under investigation, Fig. 3. Also, an increase in the extinction coefficient was observed due to the addition of In to $\mathrm{As}_{2} \mathrm{Se}_{3}$. Extinction coefficient, $\mathrm{k}$, is considered as a measure of absorption since it is directly related to the absorption coefficient, $\alpha$, through the relation:

$$
\alpha=\frac{4 \pi k}{\lambda}=2 k \frac{2 \pi}{\lambda}=2 k \frac{\omega}{C}
$$

where, $\alpha$ is the absorption coefficient, $\lambda$ is the wavelength, $\omega$ is the angular frequency and $\mathrm{C}$ is the speed of light. Spectral dependence of $\alpha$ for all films is shown in Fig. 4, where the absorption coefficient was found to increase with both incident photon energy and In content. Analysis of the strong absorption region $\left(\alpha \geqslant 10^{4} \mathrm{~cm}^{-1}\right)$ has been carried out using the following well-known quadratic equation, often called Tauc's law [15]:

$$
(\alpha h v)^{1 / 2}=B\left(h v-E_{g}\right)
$$

where B is a substance parameter (which depends on electronic transition probability, sometimes called band edge parameter) $[16,17]$ and $\mathrm{E}_{\mathrm{g}}$ is the so-called Tauc's gap. The dependence of $(\alpha \mathrm{h} v)^{1 / 2}$ on (hv) is shown in Fig. 5. It is seen that the absorption edge is shifted towards lower photon energy with the increase of indium content. The optical gap $\mathrm{E}_{\mathrm{g}}$ for non-direct transitions can be determined from interception of $(\alpha \mathrm{h} v)^{1 / 2}$ vs. (hv) with the energy axis at $(\alpha \mathrm{h} v)^{1 / 2}=0$. The good linear plot of the $(\alpha \mathrm{h} v)^{1 / 2}$ vs. (hv) indicates that the absorption mechanism in the $\operatorname{In}_{\mathrm{x}}\left(\mathrm{As}_{2} \mathrm{Se}_{3}\right)_{1-\mathrm{x}}$ films consists in non-direct transitions. The real and imaginary parts of the dielectric function, $\varepsilon^{\prime}$ and $\varepsilon^{\prime \prime}$ are related to $\mathrm{n}$ and $\mathrm{k}$ through:

$$
\varepsilon^{\prime}=n^{2}-k^{2} \text { and } \varepsilon^{\prime \prime}=2 n k
$$

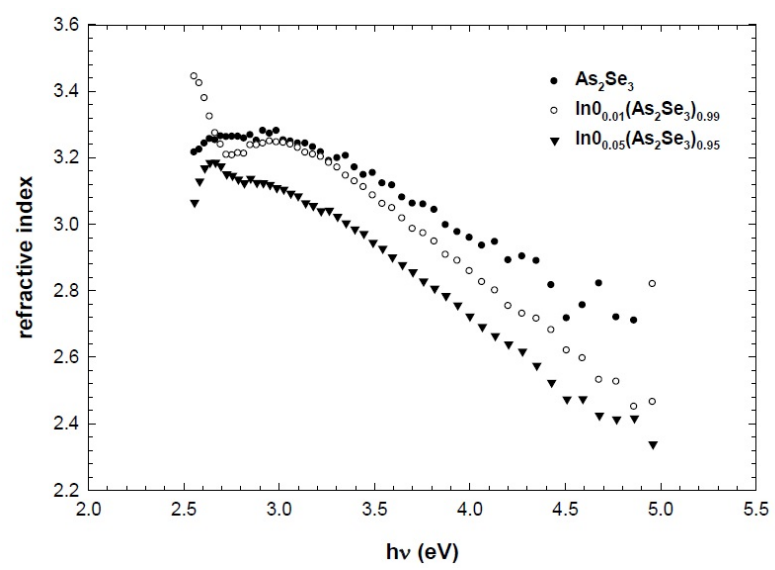

Fig. 2. Variation of refractive index with photon energy.

Variations of $\varepsilon^{\prime}$ and $\varepsilon^{\prime \prime}$ with $\mathrm{h} v$ are shown in Fig. 6 and Fig. 7. In Fig. 6, it can be observed that values of $\varepsilon^{\prime}$ decrease with the increase of In content. In Fig. 7, it can be seen that $\varepsilon^{\prime \prime}$ increases with 


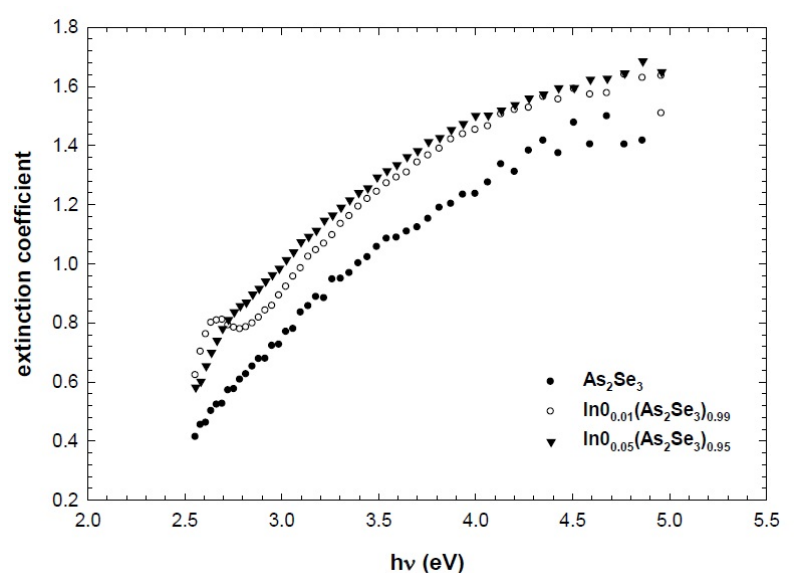

Fig. 3. Variation of extinction coefficient with photon energy.

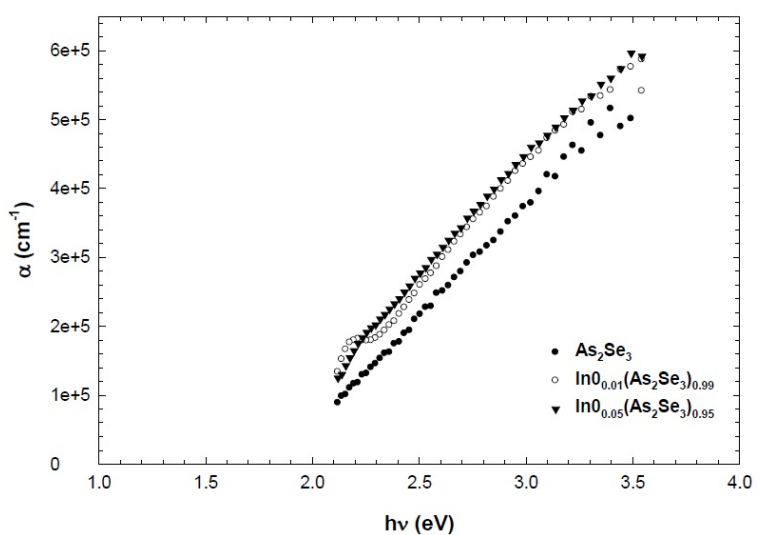

Fig. 4. Variation of absorption coefficient with photon energy.

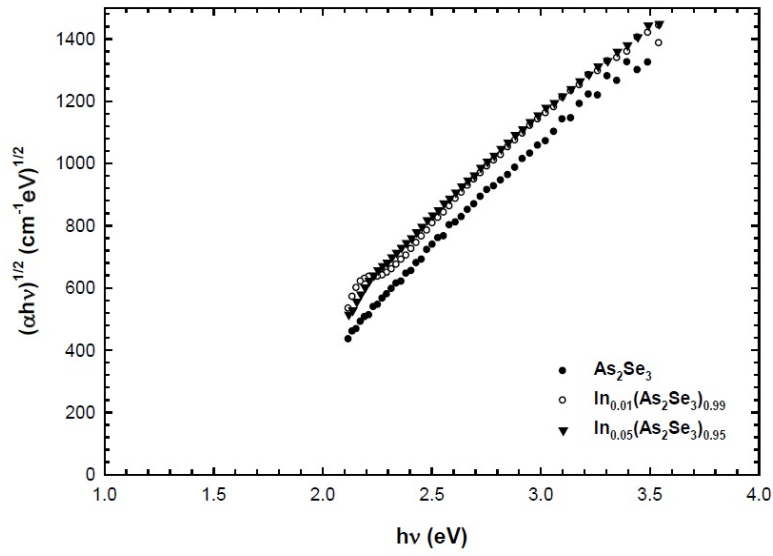

Fig. 5. Variation of $(\alpha \mathrm{h} v)^{1 / 2}$ with photon energy. increasing In content. The dissipation factor, $\tan \delta$, is expressed as:

$$
\tan \delta=\varepsilon^{\prime \prime} / \varepsilon^{\prime}
$$

Variation of dissipation factor with $\mathrm{h} v$, for all films, is shown in Fig. 8. It is clearly observed that dissipation factor increases with hv and also increases with the increase of In content.

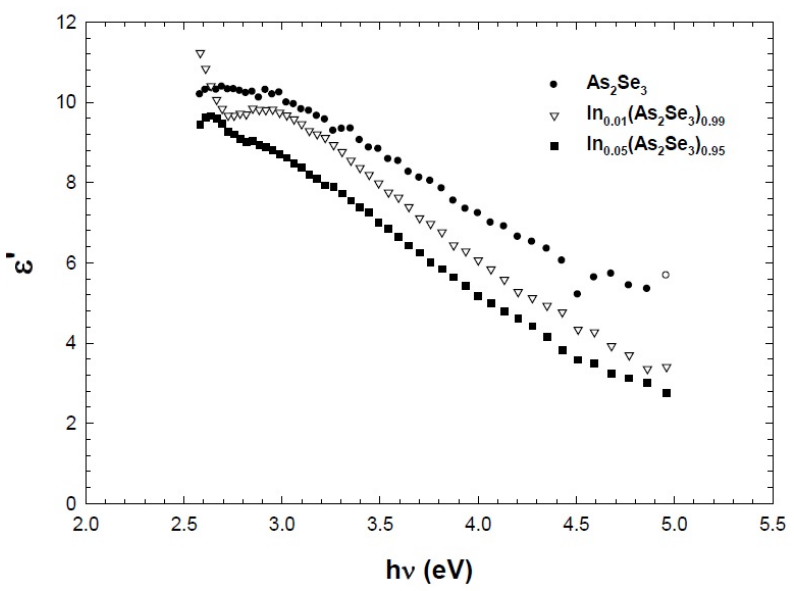

Fig. 6. Variation of the real part of the complex refractive index with photon energy.

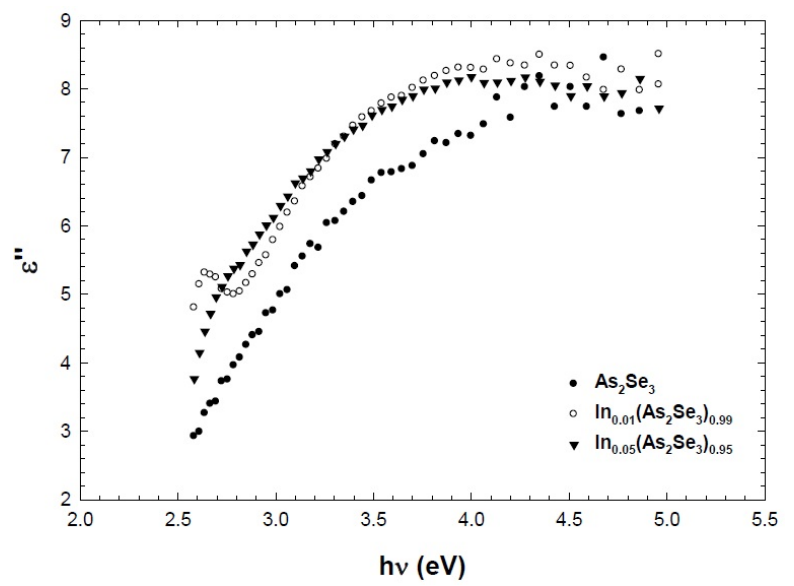

Fig. 7. Variation of the imaginary part of the complex refractive index with photon energy.

\section{Discussion}

Indium atoms are introduced into the amorphous $\mathrm{As}_{2} \mathrm{Se}_{3}$ network as impurity atoms that may 


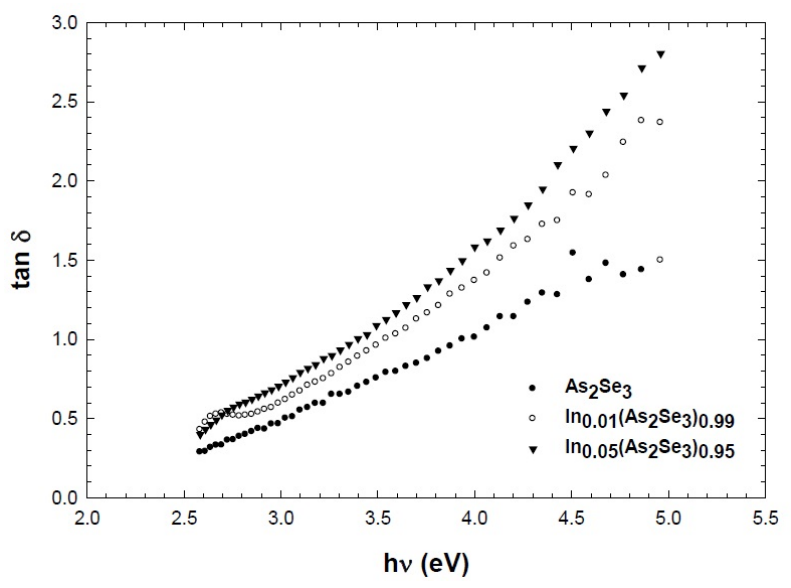

Fig. 8. Variation the dissipation factor with photon energy.

have effect on some optical parameters such as $n$ and $\mathrm{E}_{g}$. For compositions with high content of Se, there is a possibility of formation of some $\mathrm{Se}_{8}$ rings in the network. Addition of small amounts of metals such as $\mathrm{Ag}, \mathrm{Cu}, \mathrm{Tl}$, In to $\mathrm{As}_{2} \mathrm{Se}_{3}$ may modify the glassy network. Formation of ternary compounds such as $\mathrm{TlAsSe}_{2}$ and $\mathrm{AgAsSe} \mathrm{A}_{2}$ was observed at higher metal concentrations $[18,19]$. The modification of the glassy network is expressed in the form of breaking of chains and changing of the structural units of the glass former. In case of $\mathrm{As}_{2} \mathrm{Se}_{3}$, the $-\mathrm{AsSe}_{3}-\mathrm{AsSe}_{3}-\mathrm{AsSe}_{3}$ - chains are broken by metal ions and pyramidal units with one non-bridging selenium atom are created. Moreover, variation of optical parameters by adding In to $\mathrm{As}_{2} \mathrm{Se}_{3}$ can be discussed in terms of unsaturated bonds present in the composition. It is known that unsaturated bonds result from an insufficient number of atoms in deposited films. These bonds are responsible for the formation of some defects in the films. Such defects produce localized states in the band gap of the films. The presence of high concentration of localized states in the band structure is responsible for the variation of all optical parameters [20]. Consequently, metallic impurities in amorphous chalcogenides will increase disorder as a result of the increase of homopolar defect bonds at the expense of heteropolar ones. Increase of structural disorder will lead to increasing the number of localized states in the gap and hence, the depth of localized states tail in the gap [21, 22]. This will, in turn, increase the transition probabilities through localized states to the conduction band.

\section{Nonlinear optical analysis}

Nonlinear optics deals with nonlinear polarization response of a material for the incident radiation. Determination of nonlinear optical parameters of materials is helpful for the fabrication of frequency conversion and optical switching devices, which are important parts of integrated photonics. Chalcogenide glasses are highly non-linear materials ( $\chi^{(3)}$ materials) and hence, have been proposed for ultrafast switching in telecommunication systems. Their $\chi$ values are about two orders of magnitude higher than that of silica. Hence, determination of these parameters is very important for various photonic applications. Experimental determination of nonlinear optical properties $\left(\chi^{(3)}\right.$ and $\left.\mathrm{n}_{2}\right)$ requires some complicated techniques [23, 24], which are not generally available in most of laboratories. This study reports on determination of nonlinear optical parameters (third order nonlinear susceptibility $\left(\chi^{(3)}\right)$ and nonlinear refractive in$\left.\operatorname{dex}\left(\mathrm{n}_{2}\right)\right)$ from linear optical parameters $\left(\chi^{1}\right), \mathrm{n}_{0}$ and $\left.E_{g}\right)$ using semi-empirical relations [25] in the long wavelength limit. Approximate determination of $\chi^{(3)}$ can be performed using the generalized Miller's rule [26, 27]:

$$
\chi^{(3)}=A\left(\chi^{(1)}\right)^{4}
$$

where $\chi^{(1)}$ is the linear optical susceptibility and $\mathrm{A}$ is a constant having estimated value of $1.7 \times 10^{-10}$ (for $\chi^{(3)}$ in [esu]). For amorphous $\mathrm{As}_{2} \mathrm{Se}_{3}$ chalcogenide thin film, which is considered as isotropic medium, the first order approximation of the first order linear susceptibility $\left(\chi^{(1)}\right)$ is given by:

$$
\chi^{(1)}=\left(n^{2}-1\right) / 4 \pi
$$

In the long wavelength limit ( $h v \rightarrow 0$ ), this equation reduces to:

$$
\chi^{(1)}=\left(n_{0}^{2}-1\right) / 4 \pi
$$

Substituting the value of $\chi^{(1)}$ in equation 4 , the following is obtained:

$$
\chi^{(3)}=A\left(n_{0}^{2}-1\right)^{4} /(4 \pi)^{4}
$$


Table 1. Linear and nonlinear optical constants of unexposed and exposed $\operatorname{In}_{\mathrm{x}}\left(\mathrm{As}_{2} \mathrm{Se}_{3}\right)_{1-\mathrm{x}}$ thin films.

\begin{tabular}{cccccc}
\hline Composition & $\mathrm{n}_{0}(\mathrm{~h} v \rightarrow 0)$ & $\chi^{(1)}(\mathrm{h} v \rightarrow 0)[\mathrm{esu}]$ & $\chi^{(3)}(\mathrm{h} v \rightarrow 0)[\mathrm{esu}]$ & $\mathrm{n}_{2}(\mathrm{~h} v \rightarrow 0)[\mathrm{esu}]$ & $\mathrm{E}_{g}[\mathrm{eV}]$ \\
\hline \hline$\left(\mathrm{As}_{2} \mathrm{Se}_{3}\right)$ & 2.58 & 0.45 & $7 \times 10^{-12}$ & $1.7 \times 10^{-10}$ & 1.65 \\
$\mathrm{In}_{0.01}\left(\mathrm{As}_{2} \mathrm{Se}_{3}\right)_{0.99}$ & 2.63 & 0.47 & $8.4 \times 10^{-12}$ & $2.4 \times 10^{-10}$ & 1.51 \\
$\mathrm{In}_{0.05}\left(\mathrm{As}_{2} \mathrm{Se}_{3}\right)_{0.95}$ & 2.71 & 0.5 & $1.1 \times 10^{-11}$ & $2.7 \times 10^{-10}$ & 1.47 \\
\hline
\end{tabular}

The value of $\mathrm{n}_{0}$ for the undoped and doped amorphous $\mathrm{As}_{2} \mathrm{Se}_{3}$ thin films can be calculated using Wemple-DiDomenico relation [28] and the results are shown in Table 1 . In the WempleDiDomenico approach, the single-oscillator description was used to calculate the static refractive index, $\mathrm{n}_{0}$ using the equation:

$$
\varepsilon^{\prime}(\omega)=n^{2}(\omega)=1+\frac{E_{w} E_{d}}{E_{w}^{2}-(\hbar \omega)^{2}}
$$

where, $\omega$ is the frequency, $\varepsilon^{\prime}(\omega)$ is the real part of the complex electronic dielectric constant, $E_{\mathrm{w}}$ is the energy of the effective dispersion oscillator, which is defined by the mean transition energy from the valence band of the lone-pair state to the conduction band state and $E_{d}$ is the dispersion energy.

Hence, nonlinear optical susceptibility $\chi^{(3)}$, which is also shown in Table 1, can be calculated using equation 11 . The present results are found to be in good agreement with literature. The nonlinear refractive index can be determined using the semiempirical relation of Ticha et al. [24]:

$$
n_{2} \sim B / E_{g}^{4}
$$

where $\mathrm{B}=1.26 \times 10^{-9}$ [esu $(\mathrm{eV})^{4}$ ]. Putting the value of $E_{g}$, we obtain $n_{2}$ for both the doped and undoped thin films as shown in Table 1. The obtained results show good agreement with the literature. Many reports indicate that a two-photon absorption process is responsible for the optical nonlinearities observed in chalcogenide glasses. Recently, however, a multiphoton absorption process has been suggested for the nonlinearities observed in these glasses [29]. It was found that for $1.55 \mu \mathrm{m}$ radiation, the ratios of band gap energy to photon energy are 2.8, 3.4, and 4.3 for $\mathrm{As}_{2} \mathrm{~S}_{3^{-}}, \mathrm{GeS}_{2^{-}}$ and $\mathrm{TeO}_{2}$-based samples, respectively. They anticipated that the lowest-order absorption process would be three-, four-, and five-photon absorption, respectively.

\section{Conclusions}

The addition of In to the amorphous binary composition $\mathrm{As}_{2} \mathrm{Se}_{3}$ was experimentally studied using the spectroscopic ellipsometry technique. The resulting composition was found to be amorphous and no major structural changes were observed due to the addition of In. Some changes in the optical parameters calculated from the SE measurements were observed. The absorption coefficient was found to increase with the addition of In while the refractive index was found to decrease with In addition. Also, the optical energy gap, $E_{g}$, was found to decrease with the increase of In content. This may be explained as resulting from the structural changes towards increasing randomness leading to the creation of defects which consequently increases the density of localized states in the gap. This will consequently lead to an increase in transition probabilities and hence, narrowing of energy gap. Imaginary part as well as the dissipation factor of the dielectric function increased with the increase of In content. However, the real part was found to decrease with increasing In content. Also, the static refractive index $\left(\mathrm{n}_{0}\right)$ increased with increasing In content. The values of $\chi^{(3)}$ and $\mathrm{n}_{2}$ obtained from semi-empirical relations show an increment in $\chi^{(3)}$, however, the changes in $\mathrm{n}_{2}$ are negligible. All changes in linear/nonlinear optical parameters are a consequence of local structural modifications caused by the addition of In. Flexible structure and highly metastable state of amorphous chalcogenides are responsible for local structural changes caused by low concentration of metallic additives. This property of amorphous 
chalcogenides can be utilized to optimize linear/nonlinear optical parameters according to desired photonic application.

\section{References}

[1] Singh J., Shimakawa K., Advances in Amorphous Semiconductors, Taylor \& Francis, London and New York, 2003.

[2] IKeda Y., Shimakawa K., Chalcogenide Lett., 2 (2005), 127.

[3] Andriesh A.M., Iovu M.S., Shutov S.D., J. Optoelectron. Adv. Mater., 4 (2002), 631.

[4] Chen G., Jain H., Vlcek M., Ganjoo A., Phys. Rev. $B, 74$ (2006), 174203.

[5] Gotoh T., Tanaka K., J. Appl. Phys., 89 (2001), 4703.

[6] Zakery A., Elliott S.R., J. Non-Cryst. Solids, 330 (2003), 1.

[7] Bowden B.F., Harrington J.A., Appl. Optics, 48 (2009), 3050.

[8] Marquez E., Gonzalez-Leal J.M., BernalOliva A.M., WÁgNer T., JiMENEZ-Garay R., $J$. Phys. D Appl. Phys., 40 (2007), 5351.

[9] IKeda Y., ShimaKawa K., Chalcogenide Lett., 2 (2005), 125.

[10] Iovu M.S., Boolchand P., Georgiev D.G., J. Optoelectron. Adv. Mater., 7 (2005), 763.

[11] Mammadov E., Shim Y., Sakamoto J., Wakita K., Mamedov N., UchiKi H., Jpn. J. Appl. Phys., 50 (2011), 05FC12.

[12] Nelder J.A., Mead R., Comput. J., 7 (1965), 308.

[13] AzZAm R.M.A., BAshara N.M., Ellipsometry and Polarized Light, North-Holland, Amsterdam, 1986.
[14] Lippens P.E., El IdRissi Raghni M.A., OliVierFourcade J., Jumas J.C., J. Alloy. Compd., 298 (2000), 47.

[15] TAUC J., Amorphous and liquid semiconductors, Plenum Press, New York, 1974.

[16] Mott N.F., Davis E.A., Electronic Processes in NonCrystalline Materials, Oxford University Press, Oxford, 1979, p. 273.

[17] Elliot S.R., Physics of amorphous Materials, Longman, 1990.

[18] Ogusu K., Maeda S., Kitao M., Li H., Minakata M., J. Non-Cryst. Solids, 347 (2004), 159.

[19] Jenkins T.E., J. Phys. D Appl. Phys., 32 (1999), R45.

[20] El-SaYed S.M., Amin G.A.M., NDT\&E Int., 38 (2005), 113.

[21] Amin G.A.M., Spyrou N.M., Radiat. Phys. Chem., 72 (2005), 419.

[22] Sheik-Bahae M., Said A.A., Stryland E.W., Opt Lett., 14 (17) (1989), 955.

[23] Nasu H., Bara Y., Kubodera K., J. Non-Cryst Solids, $110(2-3)$ (1989), 229.

[24] Ticha H., Tichy L., J. Optoelectron. Adv. M., 4 (2) (2002), 381.

[25] Wang C.C., Phys. Rev. B, 2 (6) (1970), 2045.

[26] Wynne J.J., Phys. Rev. B, 178 (1969), 1295.

[27] Wemple S.H., Didomenico M., Phys. Rev. B, 3 (1971), 1338.

[28] Wemple S.H., Phys. Rev. B, 7 (1973), 3767.

[29] Bindra K.S., Bookey H.T., KAR A.K., WherretT B.S., Appl. Phys. Lett., 79 (2001), 1939.

Received 2014-10-09 Accepted 2015-04-29 